\title{
Saúde da população LGBT+ no contexto da atenção primária em saúde: relato de oficina realizada no internato integrado de Medicina de Família e Comunidade/Saúde Mental em uma universidade pública
}

\author{
LGBT+ Health on Primary Care: the experience report of a workshop during the medical \\ internship on Family Medicine and Mental Health in a public university
}

\section{Salud de la población LGBT + en el contexto de la atención primaria en salud: relato de taller realizado en el internado integrado de Medicina de Familia y Comunidad/Salud Mental en una universidad pública}

\begin{abstract}
Gabriela Bueno Loria1, Guilherme Martinolli Faig Canesin¹, Guilherme Martins Silva1, Gustavo Henrique de Oliveira Amorim, Julia Mendes de Melo ${ }^{1}$, Laerte Romualdo Santos ${ }^{1}$, Larissa Fonte Dutra da Rosa1, Clarisse Rinaldi Salles de Santiago ${ }^{1}$, Denise da Silva Mattos ${ }^{1}$, Michele Lopes Pedrosa', Erotildes Maria Leal'
\end{abstract}

${ }^{1}$ Universidade Federal do Rio de Janeiro. Rio de Janeiro, RJ, Brasil.

\section{Resumo}

Introdução: A saúde da população LGBT+ apresenta particularidades e vulnerabilidades que requerem atenção diferenciada. Sensibilizar e qualificar profissionais de saúde para as necessidades dessa população é fundamental para garanti-la o direito à saúde. Os currículos das graduações em saúde, que em geral não incorporam tais questões, têm sido interrogados pelo alunado com denúncias de LGBTfobia no curso médico e reivindicação de capacitação prática. Nesse contexto, o Internato Integrado de Medicina de Família e Comunidade e Saúde Mental da Faculdade de Medicina da Universidade Federal do Rio de Janeiro organizou oficina sobre Saúde da População LGBT, apresentada neste artigo. Métodos: Realizada em maio de 2018, teve como público alvo internos em estágio curricular na Atenção Primária em Saúde (APS), no município do Rio de Janeiro. Sensibilizar para o tema e apresentar ferramentas úteis para o cuidado na APS, e em outros cenários, foram os objetivos. Graduandos de medicina autodeclarados LGBT + foram convidados a assumir a condução da atividade, preparada sob orientação de professoras do internato. O protagonismo dado a esses alunos permitiu articular à expertise científica, promovida nos estudos regulares sobre o tema, a expertise experiencial. A oficina ocorreu em 4 tempos: i) sensibilização; ii) discussão de casos; iii) informação e exposição de orientações para boas práticas em saúde; iv) dúvidas e avaliação. A duração total foi de 4 horas, com metodologias ativas e participativas. Resultados: Os objetivos foram alcançados e a atividade bem avaliada em sua organização e execução. Avaliação narrativa foi realizada com alunos e professores organizadores. Os internos participantes responderam questionário online com perguntas abertas e fechadas e também avaliaram positivamente a atividade nos quesitos metodologia e conteúdo. Conclusão/Desdobramentos: A oficina foi incluída nas atividades regulares do internato. Estão em construção, com vistas a difundir esses conhecimentos a outros estudantes do curso médico e a profissionais da rede de saúde municipal, disciplina eletiva e projeto de extensão. A inclusão longitudinal do tema no currículo permanece como desafio.

Palavras-chave: Saúde das Minorias; Minorias Sexuais e de Gênero; Educação Médica; Atenção Primária à Saúde; Medicina de Família e Comunidade

Como citar: Loria GB, Canesin GMF, Silva GM, Amorim GHO, Melo JM, Santos LR, et al. Saúde da população $\mathrm{LGBT}+$ no contexto da atenção primária em saúde: relato de oficina realizada no internato integrado de Medicina de Família e Comunidade/Saúde Mental em uma universidade pública. Rev Bras Med Fam Comunidade. 2019;14(41):1807. https://doi.org/10.5712/rbmfc14(41)1807
Autor correspondente: Gabriela Bueno Loria. E-mail: gabrielaloria@gmail.com Fonte de financiamento: declaram não haver. Parecer CEP: não se aplica. Procedência e revisão por pares: revisado por pares. Recebido em: 30/06/2018. Aprovado em: 10/03/2019. 


\begin{abstract}
Introduction: The LGBT+ healthcare is marked with particularities and vulnerabilities that require differenced attention. Sensitizing and qualifying healthcare professionals to these population's needs is fundamental to guarantee the right to health. The curriculum from health degrees, that usually does not address this issue, have been questioned by students along with LGBTphobia reports among the medical education and revindication for practical capacitation. In this context, the Integrated Family \& Community Medicine (MFC) and Mental Health Internship at Faculty of Medicine of the Federal University of Rio de Janeiro, prepared a workshop on LGBT Health, presented in this article. Methods: A workshop took place in May of 2018, having a target audience of interns of MFC. It aimed to sensitize medical students regarding the referred theme and to give practical tools to be used in primary medicine and other healthcare environments. Medical undergrads who identify themselves as LGBT+ were invited to conduct the activity under the orientation of tutors from the internship. Given that those medical students were protagonists in this activity, it was possible to articulate the scientific knowledge, accomplished with regular studies on the theme, and the experiential expertise. The workshop was divided in four acts: i) sensitization; ii) case discussion; iii) information and orientations on good practice; iv) questions and feedback. The workshop lasted 4 hours, using active and participatory learning methods. Results: The objectives were achieved with a highly good evaluation as to the workshop's organization and performance. The students and professors that coordinated the activity prepared a narrative evaluation. The workshop's subjects, the MFC interns, took later an online feedback questionnaire, in which they positively evaluated the methods and acquired knowledge. Conclusion/Future works: The workshop, firstly implemented as an experimental activity, is now part of the regular medical internship curriculum. An optative course and an extension project are being planned, aiming to reach other medical students and public healthcare providers. Including the subject in the medical curriculum as a longitudinal discussion remains a challenge.
\end{abstract}

Keywords: Minority Health; Sexual and Gender Minorities; Education, Medical; Primary Health Care; Family Practice

\title{
Resumen
}

Introducción: La atención médica LGBT + está señalada con particularidades y vulnerabilidades que requieren una atención diferenciada. Sensibilizar y calificar a los profesionales de la salud de las necesidades de esta población es fundamental para garantizar su derecho a la salud. El plan de estudios de las carreras de salud, que en general no abordan este tema, ha sido cuestionado por los estudiantes junto con los informes de LGBTphobia entre la educación médica y la reivindicación de la capacitación práctica. En este contexto, la Pasantía Integrada de la Medicina de la Família y la Comunidad y Salud Mental de la graduación en medicina de la Universidad Federal de Rio de Janeiro elaboró un taller sobre salud LGBT+, presentado en este artículo. Métodos: Realizado en mayo de 2018 con los internos en pasantía curricular en la APS como el público-objetivo. Los objetivos eran sensibilizar a los estudiantes de medicina sobre el tema presentado y ofrecer herramientas prácticas para que las utilicen en la medicina primaria y en otros entornos de atención médica. Se invitó a los estudiantes de medicina que se identifican a sí mismos como LGBT+, para realizar la actividad bajo la orientación de tutores de la pasantía. Como los estudiantes de medicina fueron los protagonistas de esta actividad, fue posible articular el conocimiento científico, realizado con estudios regulares sobre el tema y la vivencia experiencial. El taller se dividió en cuatro partes: i) sensibilización; ii) discusión del caso; iii) información y orientaciones sobre buenas prácticas; iv) preguntas y comentarios. El taller tuvo una duración de cuatro horas, utilizando métodos de aprendizaje activo y participativo. Resultados: Los objetivos se lograron con una evaluación muy buena de la organización y el desempeño del taller. Los estudiantes y profesores que coordinaron la actividad realizaron una evaluación narrativa. Los pasantes presentes contestaron a un cuestionario en línea con preguntas objetivas y abiertas y evaluaron positivamente la actividad tanto por su metodología como por el contenido. Conclusión/Desarrollo: El taller, implementado como una actividad experimental, ahora forma parte del plan de estudios de prácticas médicas regulares. Se está planificando un curso optativo y un proyecto de extensión, con el objetivo de llevar eses conocimientos a otros estudiantes de medicina y proveedores de la salud pública. Incluir el tema en los planes de estudios médicos como una discusión longitudinal sigue siendo un desafío.

Palabras clave: Salud de las Minorías; Minorías Sexuales y de Género; Educación Médica; Atención Primaria de Salud; Medicina Familiar y Comunitaria

\section{Introdução}

\section{Por que discutir saúde da população LGBT+ na graduação médica?}

Os princípios doutrinários do Sistema Único de Saúde (SUS) - universalidade, integralidade e equidade - visam a garantia constitucional do direito à saúde, entendida de forma ampla e integral. Políticas públicas que identifiquem as necessidades de grupos vulneráveis, dentre os quais a população de lésbicas, gays, bissexuais, travestis e transexuais (LGBT+), contribuem para ampliar a equidade na medida em que buscam reduzir o impacto de determinantes sociais e atender demandas específicas desse grupo populacional. 
A vulnerabilidade da população LGBT+ e seu acesso precário aos serviços de saúde são desafios postos, ${ }^{1}$ apesar das recentes conquistas no âmbito das políticas públicas. ${ }^{2} \mathrm{O}$ afastamento das unidades de assistência é reflexo de diversos obstáculos como discriminação, constrangimento, preconceito, estigma e falta de treinamento profissional adequado para o atendimento a esses indivíduos. A heteronormatividade institucional, ou seja, a presunção da heterossexualidade como regra, tem efeitos deletérios sobre o acolhimento e o processo de cuidado da população LGBT+. ${ }^{3}$ Assim, a sensibilização e qualificação dos profissionais acerca das especificidades LGBT+ são passos fundamentais para garantir o efetivo cumprimento do direito à saúde, ${ }^{1}$ sendo necessário incorporar o tema aos currículos de graduação e cursos de capacitação e de educação continuada/permanente em saúde.

Rufino et al., ${ }^{4}$ entrevistaram alunos do internato em medicina de quatro faculdades localizadas no estado do Piauí, visando avaliar o ensino da sexualidade humana durante a graduação. Identificaram a predominância dos aspectos biológicos e/ou do ensino das doenças associadas à prática sexual na abordagem da temática, sendo pouco frequentes discussões sobre atendimento integral e humanizado em situações de violência sexual, aborto legal e saúde geral para lésbicas, gays, bissexuais, travestis e transexuais. De forma semelhante, estudo do currículo de uma escola médica, realizado no Rio Grande do Norte ${ }^{5}$ observou a quase ausência de abordagem de aspectos sociais e culturais da sexualidade e de matéria relativas à identidade de gênero, orientação sexual e saúde da população LGBT+, o que certamente tem impacto sobre a qualidade da atenção a ser prestada pelos egressos.

\section{A saúde da população LGBT+ na graduação em medicina da Universidade Federal do Rio de Janeiro}

O curso de graduação em medicina da universidade pública federal em foco é, como os demais cursos médicos, muito deficitário no ensino e capacitação para o trato das questões de saúde da população LGBT+. Gênero, sexualidade e diversidade são conteúdos pontuais, praticamente inexistentes, apesar da extensa carga horária curricular vigente.

Nos últimos anos, a despeito da ausência do debate sobre gênero e sexualidade no currículo formal, o alunado fez essa questão emergir por outros caminhos. No ano de 2016 foi criado um coletivo LGBT+ na Faculdade de Medicina (FM) com o propósito de acolher pessoas LGBT+ entre os próprios discentes e ajudá-las a enfrentarem a LGBTfobia em salas de aula e espaços de convivência. Paralelamente, outros fatos foram desvelando a temática LGBT+ e tornando imprescindível o seu debate: a) desmonte institucional de grupo organizado de alunos que constrangiam e oprimiam colegas com iniciativas e ações claramente racistas, machistas e LGBTfóbicas; b) denúncias por redes sociais de atitudes LGBTfóbicas praticadas por professores e funcionários técnico-administrativos; c) encontro dos alunos do internato com a população LGBT+, ao longo do internato integrado de Medicina de Família e Comunidade e Saúde Mental (internato integrado MFC/SM), realizado nas clínicas da família do município, suscitando, por parte dos estudantes, a necessidade de discussão dos problemas relativos à saúde dessa população; d) produção de trabalhos de conclusão do internato integrado MFC/SM sobre saúde da população LGBT+. Frente à conjuntura descrita, tornou-se premente a problematização da temática junto ao corpo social da faculdade de medicina e, nesse contexto, foi criada a oficina sobre a saúde da população LBGT+, objeto de relato deste artigo. 


\section{Os pressupostos e os objetivos da oficina sobre a saúde da população LGBT+ no contexto da Atenção Primária em Saúde}

Ser lésbica, gay, bissexual e transexual, ter qualquer identidade de gênero, expressão de gênero ou orientação sexual não configura doença e sim características pessoais. ${ }^{6,7}$ A gênese das condições de saúde e adoecimento da população LGBT+, quer em sua dimensão estrutural, inter-relacional ou internalizada, encontra-se na discriminação e no estigma a que ela está submetida, bem como nas diferenças e desigualdades em saúde que a afetam. ${ }^{8-12}$ Desse modo, o enfrentamento do estigma, do preconceito e da discriminação são temas centrais nos debates sobre o cuidado à saúde à população LGBT+. Esta foi a perspectiva para a elaboração de uma oficina sobre saúde da população LGBT+ visando discutir com estudantes do internato do curso médico de uma universidade pública federal boas práticas no cuidado desses indivíduos, tanto na Atenção Primária em Saúde (APS) quanto em outros cenários de atuação profissional.

O enfrentamento do estigma, do preconceito e da discriminação se tornaram temas centrais e imprescindíveis nos debates sobre o cuidado à saúde dessa população. Esses aspectos nortearam a elaboração de uma oficina sobre saúde da população LGBT+ que se propôs discutir com os internos do curso médico boas práticas no cuidado a esses indivíduos na Atenção Primária em Saúde (APS) e em outros cenários de atuação profissional.

Os alunos do internato integrado MFC/SM decidiram trazer esse debate à cena, acolhendo a proposta de uma de suas professoras, considerando o movimento em curso na graduação de medicina da universidade pública em questão e estudos que analisaram intervenções na formação médica sobre estigma e discriminação de grupos minoritários, ${ }^{13,14}$ especificamente da população LGBT+. ${ }^{15}$ Foi priorizada metodologia de ensino e aprendizagem que valorizasse a vivência de discentes LGBT+ no contato com os serviços de saúde e no próprio curso médico. Tendo essa premissa como base, foram convidados os graduandos LGBT+ da FM a participarem da organização e condução do trabalho. Outras professoras foram integradas com a tarefa de dar suporte e orientação metodológica, sendo priorizado o protagonismo dos alunos LGBT+ na coordenação e produção da atividade. A proposta foi acolhida em sua totalidade pela coordenação docente do internato integrado.

Do ponto de vista pedagógico, adotou-se como teoria educacional a Aprendizagem Situada, especificamente a Aprendizagem Corporificada, que pressupõe a interação de aspectos intelectuais, simbólicos e afetivos no processo de ensino-aprendizagem. ${ }^{16-18} \mathrm{~A}$ possibilidade de ter na organização da oficina pessoas que vivenciam seu tema central também buscou inspiração e sustentação em trabalho de Smith et al. ${ }^{19}$ Foram elencados os seguintes objetivos:

i) sensibilizar os participantes para a importância e as peculiaridades da saúde LGBT+; ii) esclarecer as dúvidas mais comuns que envolvem a temática LGBT+, com enfoque na saúde; iii) apresentar as diretrizes e políticas nacionais e internacionais, as condições de saúde mais frequentes e as ferramentas para qualificação das práticas assistenciais da população LGBT+ ; iv) buscar superar as barreiras presentes no cuidado em saúde a partir do debate acerca das boas práticas assistenciais relacionadas. 


\section{A construção da oficina}

A oficina sobre Saúde da População LGBT+ foi planejada e executada por discentes integrantes da comunidade LGBT+ da FM e professoras do departamento de MFC, contando, ainda, com a consultoria de profissionais de saúde externos à Universidade, estudiosos do tema, militantes do movimento LGBT+. Através da expertise da experiência vivida, buscou-se ampliar a capacidade de abordagem de situações cotidianas de discriminação e de estigma (estruturais, institucionais e individuais) experimentadas por pessoas LGBT+, no contexto da APS e de outros cenários de assistência.

O grupo foi composto por quinze estudantes gays, lésbicas, bissexuais ou transexuais/ transgêneros matriculados entre o terceiro e o décimo segundo períodos, dentre os quais cinco cursando o internato integrado MFC/SM. Foram realizados encontros semanais durante os meses de março e abril de 2018, nos quais se buscou instrumentalizar ativamente os discentes para atuarem como protagonistas do planejamento, da construção e da condução das oficinas, sendo responsáveis pela sua coordenação e execução. Uma inovação no mínimo ousada em instituição de currículo tradicional.

As duas professoras que participaram de toda a montagem cumpriram papel de orientadoras, colaborando com suas formações e conhecimentos didático-pedagógicos. Foi priorizado um processo de trabalho coletivo e horizontal, sobretudo no que tange à ruptura da ordem hierárquica tradicional que estabelece professores como mentores intelectuais e alunos como meros aprendizes.

O trabalho iniciou-se por uma revisão bibliográfica sobre a saúde da população LGBT+ e posterior discussão das suas peculiaridades no contexto da APS.

Oito dos alunos organizadores tinham participado como monitores no primeiro tempo de elaboração de outra oficina, no Seminário "Sexualidade e Diversidade: Atenção primária à saúde quebrando tabus", realizado na cidade de São Paulo, a convite de um dos consultores externos do grupo. Nessa oportunidade puderam se aproximar dos debates e das práticas sobre saúde LGBT+ no âmbito da medicina de família e comunidade, adquirindo conhecimentos posteriormente utilizados na concepção do presente trabalho.

Os problemas elencados para discussão durante a oficina, frente a sua prevalência e relevância na atenção primária, foram: práticas sexuais; infecções sexualmente transmissíveis (ISTs) e HIV-AIDS; saúde mental; moradores em situação de rua e acesso ao processo de transexualização. Definiu-se a seguinte dinâmica: i) sensibilização; ii) discussão de casos; iii) informação e exposição de orientações para boas práticas em saúde; iv) dúvidas e avaliação. Todo o material que compôs cada uma dessas fases foi construído e discutido conjuntamente pelos alunos-organizadores, sob supervisão das professoras-apoiadoras.

\section{A oficina sobre saúde da população LGBT+}

A oficina sobre saúde da população LGBT+ para aproximadamente 100 alunos do $10^{\circ}$, $11^{\circ}$ e $12^{\circ}$ períodos do internato integrado MFC/SM aconteceu em maio de 2018. A atividade teve quatro horas de duração, sendo reproduzida nos turnos da manhã e tarde para dois diferentes grupos constituídos por cerca de 50 internos cada. 
A primeira atividade foi a apresentação do grupo coordenador que explicitou a sua identidade LGBT+, os objetivos pretendidos e as outras etapas a serem percorridas no decorrer do turno. A seguir os participantes foram convidados a compartilhar o entendimento que tinham do tema, estimulados por três questões disparadoras: i) "Você conhece a Política Nacional de Saúde da População LGBT?"; ii) "O que você pensa quando se fala em Saúde da População LGBT?”; iii) "Que dúvidas você tem para trabalhar essa questão?". As respostas foram registradas e debatidas, com a participação livre de todos.

$\mathrm{Na}$ atividade seguinte os participantes assistiram a dois vídeos: "O "Viado" palmeirense Homossexualidade não é ofensa"20 e "O que significa LGBT?". ${ }^{21}$ O primeiro vídeo visava explicitar a questão da discriminação e estigma a que essa população se encontra cotidianamente submetida. O segundo vídeo objetivava informar sobre o significado de cada uma das letras do acrônimo LGBT. Os dois vídeos foram debatidos no início do módulo subsequente, no qual os participantes foram subdivididos em quatro pequenos grupos de 10 a 12 estudantes.

Os coordenadores dos pequenos grupos promoveram uma breve discussão inicial sobre preconceito e diversidade sexual e de gênero, com base nos vídeos assistidos. Subsequentemente foi realizada a discussão de casos clínicos: cada pequeno grupo recebeu um caso de uma das letras da sigla LGBT. Os casos clínicos foram elaborados pelos alunos-organizadores, considerando suas experiências pessoais como usuários do sistema de saúde, a prática clínica como graduandos em medicina e a bibliografia levantada.

A condução dessa etapa também ficou sob responsabilidade dos estudantes (dois alunos por cada grupo), distribuídos de forma que a identidade de gênero/orientação sexual citada no caso correspondesse à das/dos coordenadores do respectivo grupo. Cada turma contou também com uma professora responsável por dar apoio metodológico, auxiliar na dinâmica do debate e fornecer informações adicionais, se necessário.

A leitura e discussão do caso clínico aconteceu em cada um dos grupos auxiliada por perguntas norteadoras previamente elaboradas pelos alunos-organizadores, sob orientação docente. Quando todos os grupos se juntaram novamente num grande espaço comum, o produto dos debates nos pequenos grupos foi compartilhado por seus relatores coletivamente eleitos. Ao término de cada fala a plateia foi estimulada a comentar as histórias e as informações relatadas. Um resumo de cada caso e seus objetivos encontra-se descrito a seguir.

- Caso "Lésbica": mulher cisgênero, lésbica, com queixa de corrimento vaginal; faz uso abusivo de álcool.

O objetivo foi discutir a conduta heteronormativa no atendimento clínico, a abordagem da sexualidade na consulta médica, a prevenção do câncer do colo uterino e das ISTs entre mulheres que fazem sexo com mulheres, além da maior vulnerabilidade desse grupo a abuso de substâncias psicoativas.

- Caso "Gay": homem cisgênero gay, vivendo em situação de rua por ter sido expulso de casa em função de sua sexualidade, emagrecido; apesar de nunca ter mantido relações sexuais desejava realizar testagem para HIV, pois acreditava ter se contaminado ao beijar um rapaz. 
O objetivo foi conversar sobre LGBTfobia, violência e abandono, pessoas LGBT+ em situação de rua, abordagem de práticas sexuais entre homens que fazem sexo com homens, depressão entre pessoas LGBT+, prevalência de HIV e outras IST nessa população, estigma do HIV entre homens que fazem sexo com homens.

- Caso "Bissexual": homem cisgênero bissexual, com quadro de ansiedade e depressão por ter sido abandonado pela esposa quando ela descobriu sua sexualidade.

O objetivo foi debater a invisibilidade da população bissexual, sua maior vulnerabilidade a distúrbios mentais comuns e suicídio, assim como as condutas normatizadoras dos profissionais de saúde.

- Caso "Transgênero/transexual": homem transgênero homossexual, em uso irregular de hormonioterapia transexualizadora, com quadro de náuseas, dor abdominal e atraso menstrual. Faz teste de gravidez com resultado positivo.

O objetivo foi abordar orientação sexual e identidade de gênero, práticas nocivas no processo transexualizador, assim como a consulta ginecológica para homens transgênero.

Uma exposição dialogada de 30 minutos foi realizada na etapa subsequente, a fim de sintetizar as questões levantadas. Foram apresentados: os marcos políticos internacionais e nacionais que tratam das questões LGBT+, incluindo a Política Nacional de Saúde Integral de LGBT+; dados epidemiológicos sobre saúde da população LGBT+; a articulação entre acesso, preconceito e estigma; dados sobre saúde mental e condição de saúde quando esta população se encontra em situação de rua; questões relacionadas às práticas sexuais e à transexualização desassistida.

Ferramentas cuja utilização na prática profissional qualificam o atendimento à saúde desse grupo foram discutidas para cada uma dessas questões. Seguiu-se um momento de elucidação de dúvidas, também dirigida pelos alunos-organizadores, fechando essa etapa.

O último tempo da atividade consistiu no diálogo entre alunos-organizadores, internos e demais professores do internato, que foram estimulados a elencar os pontos fortes e fracos do trabalho, trazendo críticas e sugestões. Duas outras iniciativas de avaliação foram desenvolvidas posteriormente: uma avaliação narrativa, sob forma de roda de conversa, que aconteceu imediatamente após a atividade, com participação de alunos organizadores e professores apoiadores; uma avaliação através de formulário eletrônico, enviado aos alunos participantes por uma aluna-organizadora para desenvolvimento de seu trabalho de conclusão do internato em MFC/SM. As iniciativas de avaliação da oficina serão descritas a seguir.

\section{Avaliação e perspectivas futuras}

A avaliação da atividade aconteceu em três momentos distintos, com metodologias e objetivos diversos. A avaliação presencial se deu em dois tempos: ao longo da oficina, para explicitar o conhecimento prévio dos participantes sobre o tema e dúvidas existentes, e ao seu final, quando todos os participantes 
foram convidados a falar livremente sobre pontos fracos e fortes da atividade e a fazerem sugestões. Um segundo momento de avaliação ocorreu imediatamente após a atividade reunindo os organizadores, docentes e discentes, sob forma de roda de conversa, para explicitar suas experiências e os desafios enfrentados na condução da oficina.

A última etapa avaliativa foi um questionário eletrônico composto por três partes: a) identificação do respondentes (período de curso, idade e se participou do seminário da manhã ou da tarde); b) treze perguntas fechadas que avaliavam: dinâmica da atividade; capacidade da oficina dialogar com a experiência do participante; contribuição para a formação geral e prática médica; interesse e relevância da atividade; c) duas perguntas abertas: uma sobre importância da atividade a partir de uma palavra ou frase e outra solicitando críticas e sugestões.

Como relatado anteriormente neste artigo, no início da atividade os participantes foram convidados a responder três perguntas : i) "Você conhece a Política Nacional de Saúde da População LGBT?"; ii) "O que você pensa quando se fala em Saúde da População LGBT?”; iii) "Que dúvidas você tem para trabalhar essa questão?". As respostas, anônimas, foram registradas por escrito, recolhidas pelos organizadores e apresentadas aos participantes ao fim da atividade.

A primeira pergunta revelou que do total de 100 alunos respondentes apenas 3 conheciam previamente a Política Nacional de Saúde para a População LGBT. As respostas livres dadas à pergunta "O que você pensa quando se fala da saúde da população LGBT+?" foram agrupadas por tema. O tema prevalente foi "falta de preparo", seguido de "vulnerabilidade" e "dificuldade de acesso/preconceito". A análise das respostas à terceira pergunta - "Para você trabalhar esta questão [saúde da população LGBT+] que dúvidas você tem?" - agrupou as dúvidas em dois grandes campos: comunicação em saúde e conhecimento sobre problemas de saúde prevalentes para a população LGBT+.

No campo da comunicação em saúde as dúvidas ou relacionavam-se diretamente às habilidades de comunicação, tais como: "Como abordar as pessoas?" "Que palavras utilizar?" "Como não ser ofensivo?" "Que termos são bem aceitos?"; ou centravam-se na sexualidade: "Como e quando abordar diretamente a sexualidade?"; "A sexualidade deve ser tomada como questão na primeira consulta?". As respostas relacionadas ao conhecimento específico do tema "saúde da população LGBT+" expressavam dúvidas sobre a diferença entre prevalência de problemas de saúde na população em geral e nesse grupo específico; sobre a fertilização/adoção por casais homoafetivos; sobre as boas práticas e protocolos relacionados ao processo transexualizador e ainda sobre os espaços existentes no SUS para atendimentos às necessidades dessa população, dentre outras.

$\mathrm{Na}$ avaliação realizada pelos organizadores ao término da oficina foram explicitadas as barreiras enfrentadas na construção da mesma, notadamente a escassez de produção científica e a pouca divulgação do material existente, que dificultaram a busca por conhecimento sobre a temática "saúde LGBT+" e suas particularidades, assim como a preocupação que os espectadores considerassem o tema irrelevante para prática médica, ou ainda que os alunos-organizadores fossem vítimas de estigma e preconceito.

Todavia, visto a reação positiva dos alunos participantes frente à oportunidade de refletir sobre o tema proposto, percebeu-se que estas apreensões foram vencidas, seja pela busca sistemática de fontes bibliográficas qualificadas, que conferiram subsidio teórico às discussões, seja pelo esforço ativo em dialogar 
com os desafios e dificuldades práticas experimentadas na assistência a pessoas LGBT+, no cenário da APS, aproximando do cotidiano vivenciado pelos discentes em seus estágios profissionais.

A terceira etapa do processo de avaliação, realizado via resposta anônima do questionário eletrônico encaminhado, teve adesão de $43 \%$ dos participantes. Dentre os respondentes, $62,8 \%$ classificaram a experiência como excelente e $29,9 \%$ como boa. No que se refere às contribuições para a formação profissional, $74,4 \%$ consideraram-na significativa, enquanto $14 \%$ julgaram-na como tendo pouca ou nenhuma importância.

A maioria dos alunos se sentiu sensibilizada para o tema e apontou a necessidade de ampliação desse debate para períodos anteriores do curso médico. A introdução transversal desse conteúdo no ciclo clínico e a criação de disciplina regular exclusiva para abordagem das situações apresentadas também foram sugestões dadas pelos participantes. Dentre aqueles que não aprovaram a atividade destacaram-se comentários contrários à integração do conteúdo ao currículo da graduação, por considerá-lo de cunho exclusivamente "sociocultural", não relevante para o atendimento médico.

Frente aos dados acima apresentados referentes às diversas etapas avaliativas realizadas, inferimos que as finalidades propostas foram atingidas. Consideramos como ponto de destaque o protagonismo dos alunos LGBT+ na produção e condução da oficina, permitindo o diálogo entre as evidências científicas e a experiência vivida, pouco usual na formação acadêmica, e a legitimidade dos espaços de fala, ampliando o significado e o impacto subjetivo dessa atividade. A excelente recepção aos alunos-organizadores por parte dos participantes permitiu que os primeiros ocupassem uma posição afirmativa no que diz respeito às suas marcas identitárias, situação não habitual no ambiente universitário.

Os elogios e as críticas dos participantes, assim como as reflexões da equipe organizadora, revelaram um ponto em comum: a necessidade de ampliação das discussões de gênero e sexualidade na graduação em medicina, uma das condições para garantir a equidade em saúde para essa população. A partir de então, a oficina sobre saúde da população LGBT+ passou a ser atividade regular do internato integrado de MFC/ SM da FM. Nesse momento encontra-se em elaboração a disciplina eletiva "Gênero, sexualidade e saúde", com início proposto para o $2^{\circ}$ semestre de 2019. Ademais, alunos-organizadores foram convidados a falar sobre o tema para residentes de MFC e outros profissionais integrantes de equipes de saúde da família do município do Rio de Janeiro, estimulando a organização de um projeto de extensão. Espera-se que com essas iniciativas, e outros desdobramentos decorrentes, nossa FM possa contribuir para a difusão de conhecimentos e ferramentas de boas prática na assistência à saúde da população LGBT+, auxiliando na redução dos estigmas, na melhoria do acesso e na qualificação do cuidado prestado a esse grupo ainda invisível e negligenciado pela rede de assistência à saúde.

\section{Contribuição dos autores}

Nós, autores do artigo "Saúde da população LGBT+ no contexto da atenção primária em saúde: relato de oficina realizada no internato integrado de Medicina de Família e Comunidade/Saúde Mental em uma universidade pública", informamos que participamos de todas as etapas de construção do artigo acima identificado (concepção, elaboração, escrita e revisão). 


\title{
Agradecimentos
}

Agradecemos às coordenadoras do internato de Medicina de Família e Comunidade e de Saúde Mental, respectivamente Prof ${ }^{a}$ Maria Kátia Gomes e Prof ${ }^{a}$ Maria Tavares Cavalcanti, por terem acolhido a oficina "Saúde da População LGBT+" na disciplina, inserindo-a na programação curricular da mesma.

\section{Conflito de interesses}

\author{
Declaram não haver.
}

\section{Referências}

1. Mello L, Perilo M, de Braz CA, Pedrosa C. Políticas de saúde para lésbicas, gays, bissexuais, travestis e transexuais no Brasil: em busca de universalidade, integralidade e equidade. Sex Salud Soc (Rio J.) [Internet]. 2011 Dez;9:7-28 [acesso 2018 Abr 25];9:7-28. https://doi.org/10.1590/S1984-64872011000400002

2. Brasil. Ministério da Saúde, Secretaria de Gestão Estratégica e Participativa. Política Nacional de Saúde Integral de Lésbicas, Gays, Bissexuais, Travestis e Transexuais. [Internet]. Brasília (DF): Ministério da Saúde, 2013 [acesso 2018 Abr 20]. 32 p. Disponível em: http://bvsms.saude.gov.br/bvs/publicacoes/politica_nacional_saude_lesbicas_gays.pdf

3. Albuquerque GA, Garcia CL, Alves MJH, Queiroz CMHT, Adami F. Homossexualidade e o direito à saúde: um desafio para as políticas públicas de saúde no Brasil. Saúde Debate [Internet]. 2013 Jul/Set [acesso 2018 Abr 25]; 37(98):516-24. https://doi.org/10.1590/S010311042013000300015

4. Rufino AC, Madeiro AP, Girao MJBCl. O Ensino da sexualidade nos cursos médicos: a percepção de estudantes do Piauí. Rev Bras Educ Med [Internet]. 2013 [acesso 2018 Jun 23];37(2):178-85. Disponível em: http://www.scielo.br/scielo.php?pid=S0100$55022013000200004 \&$ script=sci_abstract\&tIng=pt

5. Santos GBS. Elaboração de um componente curricular sobre atenção à saúde da população LGBT em um Curso de Graduação em Medicina [dissertação] [Internet]. Rio Grande do Norte: Universidade Federal do Rio Grande do Norte; 2017 [acesso 2018 Jun 23 ]. Disponível em: https://repositorio.ufrn.br/jspui/bitstream/123456789/24326/1/GiordanoBrunoSouzaDosSantos_DISSERT.pdf

6. Reed GM, Drescher J, Krueger RB, Atalla E, Cochran SD, First MB, et al. Disorders related to sexuality and gender identity in the ICD-11: revising the ICD-10 classification based on current scientific evidence, best clinical practices, and human rights considerations. World Psychiatry [Internet]. 2016 Oct [acesso 2018 Abr 30];15(3):205-21. https://doi.org/10.1002/wps.20354

7. Cochran SD, Drescher J, Kismödi E, Giami A, García-Moreno C, Atalla E, et al. Proposed declassification of disease categories related to sexual orientation in the International Statistical Classification of Diseases and Related Health Problems (ICD-11). Bull World Health Organ [Internet]. 2014 Sep 1 [acesso 2018 Abr 30];92(9):672-9. https://doi.org/10.2471/BLT.14.135541

8. Hatzenbuehler ML, O'Cleirigh C, Mayer KH, Mimiaga MJ, Safren SA. Prospective associations between HIV-related stigma, transmission risk behaviors, and adverse mental health outcomes in men who have sex with men. Ann Behav Med [Internet] 2011 Oct [acesso 2018 Abr 30];42(2):227-34. https://doi.org/10.1007/s12160-011-9275-z

9. Hatzenbuehler ML. Structural Stigma and the Health of Lesbian, Gay, and Bisexual Populations. Curr Dir Psychol Sci [Internet]. 2014 Apr [acesso 2018 Abr 23];23(2):127-32. https://doi.org/10.1177/0963721414523775

10. Hatzenbuehler ML, Pachankis JE. Stigma and Minority Stress as Social Determinants of Health Among Lesbian, Gay, Bisexual, and Transgender Youth: Research Evidence and Clinical Implications. Pediatr Clin North Am [Internet]. 2016 Dec [acesso 2018 Jun 23];63(6):985-97. https://doi.org/10.1016/j.pcl.2016.07.003

11. Coleman S, Boehmer U, Kanaya F, Grasso C, Tan J, Bradford J. Retention Challenges for a Community-Based HIV Primary Care Clinic and Implications for intervention. AIDS Patient Care STDS [Internet]. 2007 Sep [acesso 2018 Jun 23];21(9):691-701. https://dx.doi. org/10.1089\%2Fapc.2006.0205

12. Cahill S, Valadéz R, Ibarrola S. Community-based HIV prevention interventions that combat anti-gay stigma for men who have sex with men and for transgender women. J Public Health Policy [Internet]. 2013 Jan [acesso 2018 Jun 23];34(1):69-81. https://doi.org/10.1057/ jphp.2012.59 
13. Powell Sears K. Improving cultural competence education: the utility of an intersectional framework. Med Educ [Internet]. 2012 Jun [acesso 2018 Jun 23];46(6):545-51. https://doi.org/10.1111/j.1365-2923.2011.04199.x

14. Thornicroft G, Mehta N, Clement S, Evans-Lacko S, Doherty M, Rose D, et al. Evidence for effective interventions to reduce mentalhealth-related stigma and discrimination. Lancet [Internet]. 2016 Mar [acesso 2018 Jun 23];387(10023):1123-32. https://doi.org/10.1016/ S0140-6736(15)00298-6

15. Fallin-Bennett K. Implicit bias against sexual minorities in medicine: cycles of professional influence and the role of the hidden curriculum. Acad Med [Internet]. 2015 May [acesso 2018 Jun 24];90(5):549-52. https://doi.org/10.1097/ACM.0000000000000662

16. Lave J. Cognition in Practice: Mind, mathematics, and culture in everyday life. Cambridge: Cambridge University Press; 1988.

17. Lave J, Wenger E. Situated Learning: Legitimate Peripheral Participation. Cambridge: Cambridge University Press; 1991.

18. McKenzie C, Barndt D, Butterwick S, Clover D, Ng R. Popular Education and Embodied Learning: Intersections for Critical and Anti-Racist Feminist Praxis. In: The 25th Annual Conference; Proceedings of the Canadian Association for the Study of Adult Education (CASAE); May 2006; Toronto, Ontario, Canada.

19. Smith G, Hughes J, Greenhalgh T. Patients as Teachers and Mentors. In: Greenhalgh T, Humphrey C, Woodard F, eds. User Involvement in Health Care. New York: John Wiley \& Sons; 2010. p. 52-63.

20.Santiago S. O "Viado" palmeirense - Homossexualidade não é ofensa [Internet]. 12 de março de 2018 [consultado em 23 de abril de 2018] [6:35min]. Disponível em https://www.youtube.com/watch?v=nszqQU9m7S8

21. Associação Lambda. O que significa LGBT? [Internet]. 8 de agosto de 2017. [consultado em 23 de abril de 2018] [0:40]. Disponível em https://www.youtube.com/watch?v=Ncg4tRMTWAY 